\title{
Physiological Studies on Floridean Starch, Floridoside and. Trehalose in a Red Alga, Serraticardia maxima*
}

\author{
by Hideyuki NAGASHIMA,** Hachiro OzAKI,*** Saheye \\ NAKAMURA**** and Kazutosi NISIZAWA****
}

Received November 11, 1969

\begin{abstract}
When a calcareous red alga, Serraticardia maxima (Ohoshikoro) was illuminated in sea water containing $\mathrm{H}^{14} \mathrm{CO}_{3}^{-}$, floridoside, floridean starch and trehalose that are major soluble carbohydrates in this alga were rapidly labelled in this $९$ der. During the dark culture following the illumination in sea water free of radiocarbon, the radioactivities and the absolute quantities of floridoside and trehalose remained almost unchanged while those of floridean starch were markedly decreased. On the other hand, feeding experiments with ${ }^{14} \mathrm{C}$-floridoside and ${ }^{14} \mathrm{C}$-trehalose on chopped algal fronds revealed that these carbohydrates were rapidly interconvertible in the intact cells. Moreover, the analysis of $\mathrm{Ca}^{14} \mathrm{CO}_{3}$ deposited in cell wall during the feeding of the fronds with ${ }^{14} \mathrm{C}$-floridoside and ${ }^{14} \mathrm{C}$-trehalose indicated that these carbohydrates were rapidly consumed by respiration of the cells. From these results, it was concluded that these carbohydrates are storage products and in particular, floridoside and floridean starch seem to play similar physiological roles to sucrose and starch in higher plants, respectively. A small amount of laminitol was foundin this red alga, but the cyclitol appears to be an inert product.
\end{abstract}

It has been known that marine algae contain a variety of carbohydrates that are rare in higher plants and moreover, their photosynthetic products are characteristic of each phylum ${ }^{1,2}$. However, Bean and Hassid ${ }^{3)}$ have reported that in a red alga, Iridophycus flaccidum, ${ }^{14} \mathrm{C}$-phosphoglyceric acid may be the first stable photosynthetic product from ${ }^{14} \mathrm{CO}_{2}$, and that most of sugar phosphates produced during photosynthesis appear to be identical with those in green plants. These authors also suggested that floridoside in this alga is a reserve carbohydrate analogous to sucrose in higher plants.

Recently, trehalose or isofloridoside was also shown to be produced at the early stages of photosynthesis in some red algae ${ }^{4,5)}$, whereas floridean starch which is a storage product and is similar to amylopectin in its structure has been found in many red algae ${ }^{6,7)}$. However, it is not clear what physiological relationships exist

* Contribution No. 196 from the Shimoda Marine Biological Station, Tokyo Kyoiku University. This work was supported by a Grant-in-aid of Cooperative Research from the Ministry of Education, Japan.

** Present Address: Department of Biology, Faculty of Science, Science University of Tokyo, Kagurazaka, Tokyo, Japan.

*** Present Address: Central Research Laboratory of Ajinomoto Co. Inc., Kawasaki,. Kanagawa, Japan.

**** Botanical Institute, Faculty of Science, Tokyo Kyoiku University, Otsuka, Tokyo, Japan. 
among these carbohydrates. Thus, it seems worth while to investigate the roles of these carbohydrates, especially in relation to the photosynthetic carbon reduction cycle. The authors attempted, therefore, to elucidate this problem using Serraticardia maxima (Ohoshikoro) as the experimental material, since it has been found by them ${ }^{8)}$ that floridoside and trehalose are present beside floridean starch in considerable amounts in this calcareous red alga.

\section{Materials and Methods}

1) Plant materials.

Fronds of Serraticardia maxima (formerly called Joculator maximus) were collected at the rocky shores of Shimoda Bay, and immediately transported to the laboratory of the Marine Biological Station. Algal frounds at a similar growth stage were selected as experimental materials, and carefully removed from contaminants sticked to the fronds. They were then cultured in running sea water for about 6 hours in the dark and used for experiments.

2) ${ }^{14} \mathrm{CO}_{2}$-assimilation in the light and in the subsequent dark culture.

Photosynthetic experiments were conducted at $16^{\circ}$ in a culture vessel with 2 liter of filtered sea water containing $2 \mathrm{mC}$ of $\mathrm{NaH}^{14} \mathrm{CO}_{3}$. The culture medium was adjusted to $\mathrm{pH}$ 8.2. Several batches of algal fronds of approximately $5 \mathrm{~g}$ fresh weight were placed each in a nylon net and shaken slowly in the vessel. A 500 watt spot-lamp was set at a distance of $50 \mathrm{~cm}$ above the vessel, and a screen through which cooled water was allowed to run was placed between them. Thus the light intensity of 5,000 lux was supplied at the surface of the culture medium. At intervals, two batches of the fronds were removed at a time, quickly washed with water and boiled with $100 \mathrm{~m} l$ of $80 \%$ ethanol for $15 \mathrm{~min}$.

After 3 hour-illumination, all the remaining batches were taken out from the culture medium, washed with water and transferred quickly into an other vessel containing running sea water free of radiocarbon, and cultured in the dark at $18^{\circ}$ for 24 hours. During this culture, two batches of the fronds in another series of experiment were simultaneously removed at intervals and treated in the same way as above.

\section{3) Preparation of each fraction.}

The algal fronds boiled with $80 \%$ ethanol for 15 min were dried in an oven at $90^{\circ}$ for 4 hours and weighed after further drying over calcium chloride in a vacuum desiccator for 5 hours. The fronds were then ground to powder in a mortar and extracted with $30 \mathrm{ml}$ of boiling $80 \%$ ethanol for $15 \mathrm{~min}$. The same treatment was repeated five times more, and the extracts were combined for further fractionation, as shown in Fig. 1.

a) Ether soluble fraction

The combined ethanol soluble fraction of about $200 \mathrm{ml}$ was concentrated to a syrup in vacuo below $40^{\circ}$, and $50 \mathrm{ml}$ of ether was added. After the mixture was sufficiently shaken, ether soluble fraction was separated from the syrup. This fraction contained pigments such as chlorophyll, phenolic compounds and lipids, and it was referred to as "ether soluble fraction".

b) Neutral sugar fraction

The remaining syrup from the "ether soluble fraction" was then diluted with 
$50 \mathrm{ml}$ of water. The aqueous solution was deionized by passing through an Amberlite IR-120 $\left(\mathrm{H}^{+}\right)$column $(1 \times 10 \mathrm{~cm})$ and Ambrlite IRA- $410\left(\mathrm{HCOO}^{-}\right)$column $(1 \times 10 \mathrm{~cm})$. successively. The neutral effluents contained various sorts of sugars, and it was. named "neutral sugar fraction", and further fractionated by paper chromatography.

c) Amino acid and organic acid fractions

Substances adsorbed on the Amberlite IR-120 column were eluted with $80 \mathrm{ml}$ of $2 \mathrm{~N}$ ammonium hydroxide. The eluate contained various kinds of amino acids, and it was named "amino acid fraction". In the same manner, substances adsorbed on the Amberlite IRA-410 column were eluted with $80 \mathrm{ml}$ of $5 \mathrm{~N}$ formic acid. The: eluate contained various kinds of organic acids, and it was named "organic acid fraction". Sugar phosphates also may be contained in this fraction together with the organic acids.

d) Polysaccharide fraction, calcium carbonate and crude fiber

Dilute hydrochloric acid was added to the residual material from the first ethanol extraction of algal fronds to decompose $\mathrm{CaCO}_{3}$ embeded in it. ${ }^{14} \mathrm{CO}_{2}$ released from $\mathrm{Ca}^{14} \mathrm{CO}_{3}$ was then converted into $\mathrm{BaCO}_{3}$ and determined by the method as will be described later.

After decomposition of $\mathrm{CaCO}_{3}$, residual material named "polysaccharide fraction" was hydrolyzed with $1 \mathrm{~N}$ hydrochloric acid at $100^{\circ}$ for 4 hours. This acid treatment still left fibrous material, and this was named "crude fiber". The filtrate removed from the "crude fiber" was then deionized with both kinds of ion exchange resins

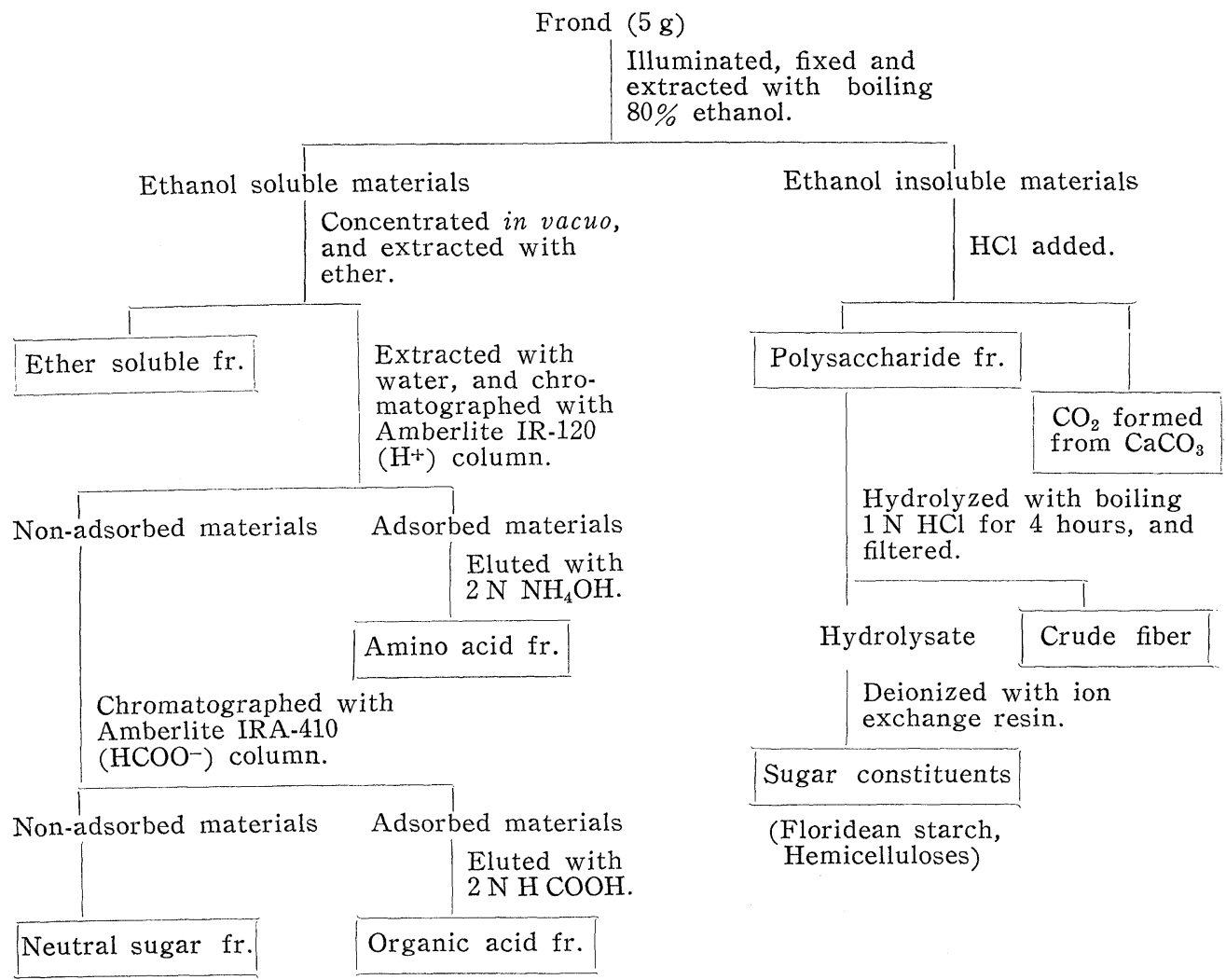

Fig. 1. Fractionation of the constituents of algal fronds after illumination. 
and concentrated in vacuo to a definite volume. The compounds therein consisted of various sugars, and they were quantitatively determined after separation by paper chromatography.

4) Analysis of the sugar components.

An aliquot of " neutral sugar fraction" was applied to Tokyo Roshi No. 50 filter paper as a band and developed with a solvent system, butanol-pyridine-water $6: 4$ : $3, \mathrm{v} / \mathrm{v})$, by ascending technique. With the aid of guide strips, it became evident that each zone contained a sugar component corresponding to any of floridoside, trehalose, laminitol, and unknown compound. Each zone was then cut off from the filter paper and sugar component was extracted therefrom with a given volume of water. After an aliquot of the extract was dried on planchet, the radioactivity of the resulting material was measured. On the other hand, amount of the sugars was determined by phenol sulfuric acid method ${ }^{9}$.

Deionized acid hydrolysate from "polysaccharide fraction" was similarly separated by band-chromatography with a solvent system, ethylacetate-pyridine-water $(10: 4: 3, \mathrm{v} / \mathrm{v})$, by ascending technique. Each zone contained galactose, glucose and xylose, and the zones were cut off each from the filter paper, and eluted with a given volume of water. Reducing sugar in the eluate was determined by Nelson-Somogyi's $\operatorname{method}^{10)}$. On the other hand, the radioactivity of these sugars was measured by the same method as described above.

5) Feeding experiments with ${ }^{14} \mathrm{C}$-carbohydrates.

a) Preparation of ${ }^{14} \mathrm{C}$-floridoside and ${ }^{14} \mathrm{C}$-trehalose

About $500 \mathrm{~g}$ of fresh fronds was illuminated at $20^{\circ}$ with 10,000 lux in 5 liters of sea water ( $\mathrm{pH} 8.2$ ) containing $5 \mathrm{mC}$ of $\mathrm{NaH}^{14} \mathrm{CO}_{3}$ for 3 hours. The fronds were then extracted several times with boiling $80 \%$ ethanol and the combined extract was concentrated to a syrup in vacuo below $40^{\circ}$. The syrup was partitioned with a small amount of ether and water, and the latter was treated with anion and cation exchange resins. The resulting salt-free solution was then chromatographed on carbon and cellulose columns successively as described in a previous paper ${ }^{8)}$, and the crude preparations of floridoside and trehalose were obtained. These compounds were recrystallized from ethanol. Yields: $645 \mathrm{mg}$ for the former and $193 \mathrm{mg}$ for the latter. Specific radioactivities: $7.88 \times 10^{4} \mathrm{cpm} / \mathrm{mg}$ and $4.20 \times 10^{4} \mathrm{cpm} / \mathrm{mg}$ for ${ }^{14} \mathrm{C}$ floridoside and ${ }^{14} \mathrm{C}$-trehalose, respectively.

b) Preparation of ${ }^{14} \mathrm{C}$-glycerol

For the preparation of ${ }^{14} \mathrm{C}$-glycerol, ${ }^{14} \mathrm{C}$-floridoside of $20 \mathrm{mg}$ was hydrolyzed with $1 \mathrm{~N}$ hydrochloric acid at $100^{\circ}$ for 1 hour, and the hydrolysate was deionized by Amberlite IRA-410 $\left(\mathrm{OH}^{-}\right)$column chromatography. The resulting solution was then subjected to band-paper chromatography to separate ${ }^{14} \mathrm{C}$-glycerol. The zone containing ${ }^{14} \mathrm{C}$-glycerol was cut off from the filter paper and extracted with water. Yield: $5.8 \mathrm{mg}$. Specific radioactivity : $6.75 \times 10^{4} \mathrm{cpm} / \mathrm{mg}$.

c) Feeding experiments

After freshly collected fronds were cultured with running sea water in darkness for 12 hours, they were cut into pieces of about $1 \mathrm{~cm}$ long. About $1 \mathrm{~g}$ of these fronds was wrapped with a nylon net in a flask containing $15 \mathrm{~m} l$ of filtered fresh sea water ( $\mathrm{pH} 7.6)$, in which one of the ${ }^{14} \mathrm{C}$-carbohydrates obtained above was dissolved. The flask was evacuated to make the infiltration of the ${ }^{14} \mathrm{C}$-carbohydrate into the fronds much easier. After $10 \mathrm{~min}$, the air pressure in the flask was re- 
covered and the flask was stirred automatically in the light under 5,000 lux or in the dark at $25^{\circ}$ for appropriate periods. The fronds were then removed from the feeding medium together with the nylon net, immediately washed with water, and boiled with $80 \%$ ethanol for 10 min. Afterr epeating the extraction with $80 \%$ ethanol three times more, the combined extract was fractionated in regard to neutral sugar constituents in the similar manner to those in the experiment of ${ }^{14} \mathrm{CO}_{2}$-assimilation. This fraction was further fractionated into individual sugars and they were identified by paper chromatography.

To $50 \mathrm{mg}$ of ethanol insoluole fraction obtained above, $1 \mathrm{~m} l$ of $1 \mathrm{~N}$ hydrochloric acid was added to decompose $\mathrm{CaCO}_{3}$. Water-soluble polysaccharide that consisted mainly of floridean starch was then precipitated by the addition of ethanol to a final concentration of $70 \%$, washed twice with ethanol, and its radioactivity was determined. Part of the polysaccharide fraction was hydrolyzed with $1 \mathrm{~N}$ hydrochloric acid and the radioactivity of hydrolysis products was measured in the same way.

6) Measurement of radioactivity.

Radioactivities of samples were determined with an Aloka TDC-1 gas flow counter. In most cases, radioactive spots or bands on paper chromatogram were first located by a model PCS-2 paper chromatogram scanner, and then cut off from the filter paper. The radioactive substances were extracted therefrom with water. When these substances were neutral sugars, an aliquot of the extracts was placed on an aluminum planchet, dried by heating and counted. In contrast, other substances such as amino acid, organic acid fractions were counted without further treatment.

Radioactivity of $\mathrm{CaCO}_{3}$ was counted by the method of Peters ${ }^{11)}$ as follows. Dilute hydrochloric acid was carefully added to $50 \mathrm{mg}$ of ethanol insoluble fraction. The carbon dioxide gas evolved therefrom was absorbed in a barium hydroxide solution. The resulting barium carbonate precipitate was collected through filter paper, and its radioactivity was measured directly on an aluminum planchet. Since self absorption of the precipitate was high, correction was made for this value.

\section{Results}

1) Photoassimilation of $\mathrm{H}^{14} \mathrm{CO}_{3}^{-}$into various cell constituents and the fates of the radioactivities during the subsequent dark culture.

a) Radioactivities of individual fractions

Fresh algal fronds were subjected to the photoassimilation of $\mathrm{H}^{14} \mathrm{CO}_{3}^{-}$for 3 hours, and the incorporation of the radioactivity into various constituents of the fronds were fractionated in the manner as shown in Fig. 1, and changes in their activities during the subsequent dark culture were examined. The results are shown in Table 1.

It is clear that the radioactivity was most rapidly incorporated into soluble neutral sugars in the light within 1 hour, and it amounted about $40 \%$ of the total radioactivity incorporated. After 3 hour-illumination, however, incorporation of the radioactivity into the polysaccharide fraction whose main constituent may be floridean starch has been gradually increased. The total incorporation into calcium carbonate was also remarkable, and it is almost equal to that of neutral sugar fraction. In contrast, the radioactivity in amino acid, organic acid and ether solu- 
Table 1. Incorporation of radioactivity into various substances during photosynthesis in $\mathrm{H}^{14} \mathrm{CO}_{3}^{-}$and changes in the radioactivities during the subsequent dark culture without $\mathrm{H}^{14} \mathrm{CO}_{3}^{-}$.

\begin{tabular}{|c|c|c|c|c|c|}
\hline \multirow{3}{*}{ Fraction } & \multicolumn{5}{|c|}{ Radioactivity, $\operatorname{cpm} \times 10^{-3} / \mathrm{g}$ dry $\mathrm{wt}$. of frond } \\
\hline & \multicolumn{2}{|c|}{ Illumination period (hr.) } & \multicolumn{3}{|c|}{$\begin{array}{l}\text { Dark-period subsequent to } \\
3 \text { hour-illumination (hr.) }\end{array}$} \\
\hline & 1 & 3 & 6 & 12 & 23 \\
\hline \multicolumn{6}{|l|}{ Ethanol soluble fraction } \\
\hline Amino acid & 191 & 557 & 490 & 443 & 364 \\
\hline Organic acid & 209 & 312 & 403 & 261 & 378 \\
\hline Neutral sugar & 2460 & 5450 & 5090 & 4770 & 4060 \\
\hline Ether soluble substance & 213 & 399 & 653 & 411 & 639 \\
\hline \multicolumn{6}{|l|}{ Ethanol insolnble fraction } \\
\hline Polysaccharide & 652 & 3240 & 2700 & 1700 & 858 \\
\hline Crude fiber & 29 & 126 & 184 & 242 & 260 \\
\hline $\mathrm{CaCO}_{3}$ & 2380 & 5540 & 4480 & 4150 & 4740 \\
\hline Total & 6130 & 15620 & 14000 & 11980 & 10300 \\
\hline
\end{tabular}

ble fraction was very low; none of them exceeded $10 \%$ of the total incorporation.

During dark culture following the illumination, the radioactivity of polysaccharide fraction was decreased markedly, but those of soluble neutral sugar fraction and calcium carbonate fraction was not. Therefore, further fractionation of the soluble neutral carbohydrates were carried out by paper chromatography to examine whether all of the neutral sugar components do not decrease even in the dark or some of them decrease but others increase to compensate as a whole.

b) Radioactivities of floridean starch, floridoside and trehalose

Since the soluble neutral sugars were fractionated mainly into floridoside, trehalose and laminitol, but the radioactivities are very high only in the former two, changes in the radioactivities in these two were followed. In parallel, changes in the radioactivity of floridean starch are also investigated. These results are

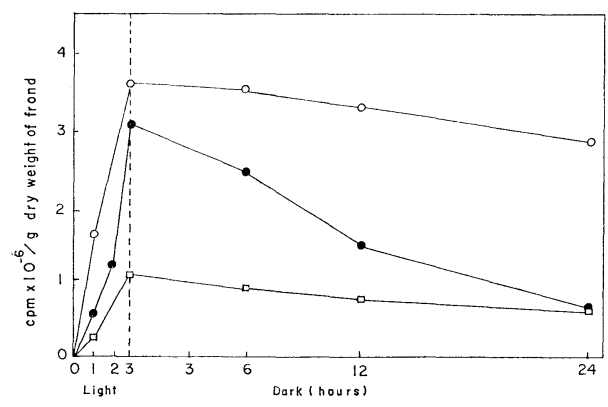

Fig. 2. Changes in radioactivities of the main carbohydrates during culture with $\mathrm{H}^{14} \mathrm{CO}_{3}^{-}$in the light and during the subsequent dark culture without $\mathrm{H}^{14} \mathrm{CO}_{3}^{-}$.

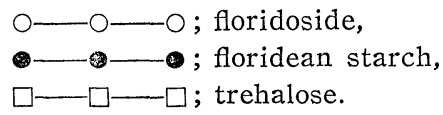
shown in Fig. 2.

It is clear in the figure that the radioactivity was most rapidly incorporated into floridoside in the light, but this radioactivity decreased in the subsequent dark culture at a slow rate as that of trehalose. In contrast, the radioactivity of starch was most rapidly decreased in the dark culture. The result seems as if floridean starch may be a readily convertible storage product.

This fact is also confirmed by the following results. As shown in Fig. 3, the absolute content of floridean starch was most abundant and increased rather rapidly in the light, and decreased rather faster than floridoside and trehalose in the dark. Moreover, the specific radioactivities of the 
three carbohydrates were equally increased in the light, while only that of floridean starch markedly decreased during the subsequent dark culture, as shown in Table 2. These results raise the question whether floridoside and trehalose would be even synthesized in the dark. In this respect only, floridean starch seems more rapidly metabolizable product than the other two.

c) Radioactivities of sugar constituents in polysaccharide fraction

Polysaccharide fraction obtained by the procedure in Fig. 1 was hydrolyzed with $1 \mathrm{~N}$ hydrochloric acid to identify and estimate approximate amounts of sugar constituents. The results are shown in

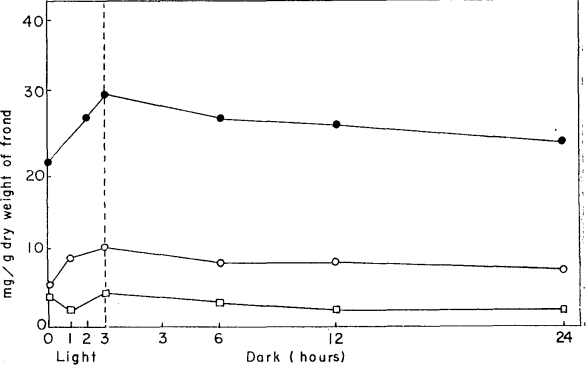

Fig. 3. Changes in the amounts of three carbohydrates in the light and in the subsequent dark cultures.

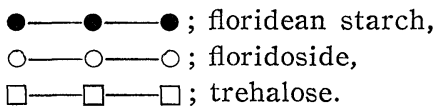
Trble 3. The polysaccharide fraction was stained with iodine in reddish purple. However, the residue after acid hydrolysis, "crude fiber", was not stained with iodine in such a color. The major constituent in the polysaccharide fraction, therefore, must be floridean starch, because it was extracted with alkali and produced maltose on $\beta$-amylolysis.

"Crude fiber" showed a slightly positive reaction with ninhydrin reagent, but it gave cellobiose and glucose upon digestion with a cellulase preparation from Trichodema viride. The main const ituent of the "crude fiber" therefore may be cellulose which is contaminated with a small amount of protein.

As other sugar constituents, galactose and xylose were detected in the polysaccharide fraction. They may be derived from galactan and xylan, respectively. In

Table 2. Change in the specific radioactivities of three carbohyrates.

\begin{tabular}{|c|c|c|c|c|c|}
\hline & \multicolumn{5}{|c|}{ Specific activity $\left(\mathrm{cpm} / \mathrm{mg} \times 10^{-3}\right)$} \\
\hline & \multicolumn{2}{|c|}{ Light-period (hr) } & \multicolumn{3}{|c|}{$\begin{array}{l}\text { Dark-period subsequent to } \\
3 \text { hour-illumination (hr) }\end{array}$} \\
\hline & 1 & 3 & 6 & 12 & 24 \\
\hline Floridoside & 173.2 & 354.3 & 429.1 & 396.9 & 374.5 \\
\hline Trehalose & 116.8 & 262.7 & 323.8 & 385.0 & 336.5 \\
\hline Floridean starch & 57.5 & 101.3 & 92.1 & 56.2 & 26.6 \\
\hline
\end{tabular}

Table 3. Amounts of the constituent sugars in polysaccharide fraction.

\begin{tabular}{lcc}
\hline Sugar constituent & \multicolumn{2}{c}{ Amount, } \\
Glucose & $\mathrm{mg} / \mathrm{g}$ dryt. of & frond \\
\hline Galactose & 27.0 & 27.4 \\
Xylose & 17.2 & 17.4 \\
Crude fiber & 14.8 & 15.0 \\
\hline
\end{tabular}




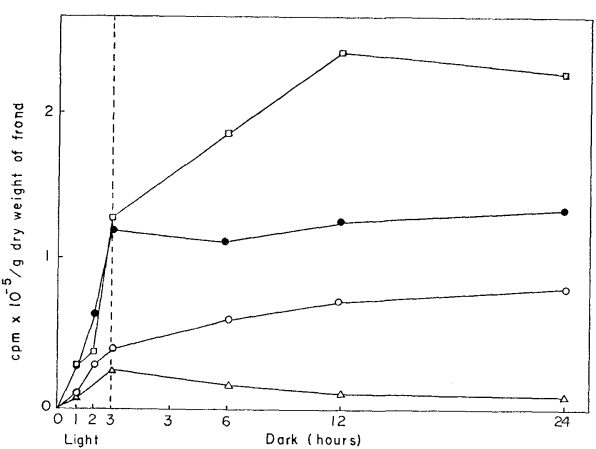

Fig. 4. Changes in radioactivities of several polysaccharides and laminitol during culture with $\mathrm{H}^{14} \mathrm{CO}_{3}^{-}$in the light and the subsequent dark culture without $\mathrm{H}^{14} \mathrm{CO}_{3}^{-}$.

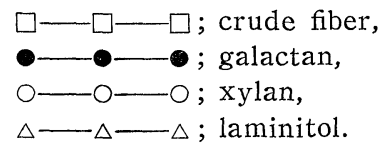

this connection, it is to be noted that Turvey ${ }^{12}$. has reported that sulfated galactan is present in the calcareous red alga, Corallina officinalis in association with xylan.

Fig. 4 shows the patterns of changes in the radioactivities of these polysaccharides beside laminitol during illumination and the subsequent dark culture. Incorporation of radiocarbon into isofloridoside was very small as. compared with that into floridoside, although not shown in the present work. It attains. only about $1-4 \%$ of the total radioactivity incorporated under the same condition (see Table 4).

d) Changes in the precent radioactivities of various compounds during light and the subsequent dark culture

Table 4 shows changes in the percentage: of radioactivity of individual carbohydrates. under illumination in the presence of $\mathrm{H}^{14} \mathrm{CO}_{3}^{-}$ and during the following dark culture. It is to be noticed that the percent radioactivity of floridoside was slightly decreased during the light periods, but it was. gradually increased up to about $60 \%$ of the total radioactivity of carbohydrates in the course of the following dark period. In contrast, the percent radioactivity of florideah starch was changed inversely. From these data only, floridean starch appears more rapidly metabolizale product than the other two.

2) Feeding experiments with ${ }^{14} \mathrm{C}$-floridoside, ${ }^{14} \mathrm{C}$-trehalose and ${ }^{14} \mathrm{C}$-glycerol.

In order to elucidate the metabolic mutual relationship between floridoside, trehalose and floridean starch, algal fronds were cut into small pieces and fed with each of the three ${ }^{14} \mathrm{C}$-labelled compounds. ${ }^{14} \mathrm{C}$-Floridoside, ${ }^{14} \mathrm{C}$-trehalose or ${ }^{14} \mathrm{C}$-glycerol was dissolved in a culture medium at a concentration indicated in the figure and allowed to be infiltrated into the algal fronds by evacuation technique.

Table 4. Changes in percent radioactivities of various carbohydrates during light culture with $\mathrm{H}^{14} \mathrm{CO}_{3}^{-}$and the subsequent dark culture without $\mathrm{H}^{14} \mathrm{CO}_{3}^{-}$.

\begin{tabular}{|c|c|c|c|c|c|}
\hline & \multicolumn{2}{|c|}{ Illumination-period ( $h r$ ) } & \multicolumn{3}{|c|}{$\begin{array}{l}\text { Dark-period subsequent to } \\
3 \text { hour-illumination (hr) }\end{array}$} \\
\hline & 1 & 3 & 6 & 12 & 24 \\
\hline Floridoside & 63.9 & 45.1 & 48.3 & 55.0 & 63.6 \\
\hline Floridean starch & 23.9 & 38.0 & 34.1 & 24.7 & 14.4 \\
\hline Trehalose & 9.2 & 12.9 & 12.6 & 12.7 & 13.6 \\
\hline Laminitol & 0.2 & 0.3 & 0.2 & 0.2 & 0.2 \\
\hline Crude fibre & 1.2 & 1.6 & 2.5 & 4.0 & 3.5 \\
\hline Galactan & 1.1 & 1.6 & 1.5 & 2.2 & 2.9 \\
\hline Xylan & 0.5 & 0.5 & 0.8 & 1.2 & 1.8 \\
\hline Total & 100.0 & 1000.0 & 100.0 & 100.0 & 100.0 \\
\hline
\end{tabular}


Fig. 5 shows the extents of radiocarbon incorporation from ${ }^{14} \mathrm{C}$-floridoside into floridean starch and trehalose of the algal fronds in the light or in the dark. Radioactivity from ${ }^{14} \mathrm{C}$-floridoside was rapidly incorporated into floridean starch both in the light and in the dark. However, trehalose was labelled only in the light, although an apparent rate of the incorporation was much slower than starch.

Fig. 6 shows the extents of radiocarbon incorporation from ${ }^{14} \mathrm{C}$-trehalose into floridean starch and floridoside of the algal fronds in the light or in the dark. In this case also, the results were similar to the above. Radiocarbon of trehalose was incorporated into floridean starch both in the light and in the dark, but not into floridoside in the dark. An apparent rate of the incorporation into starch was also much greater than floridoside.
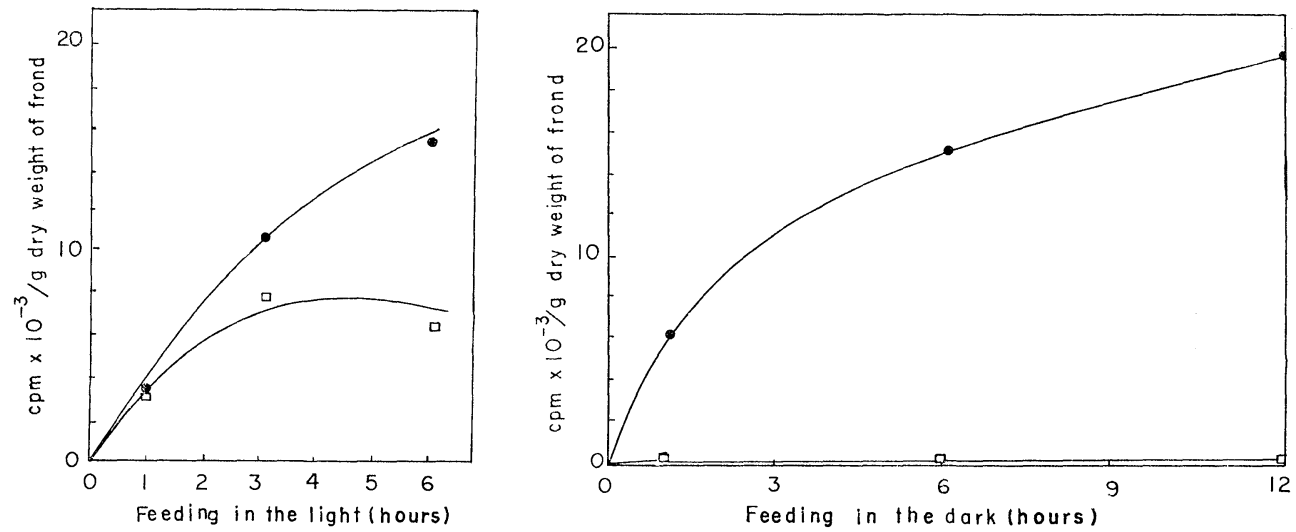

Fig. 5. Incorporation of radiocarbon into a floridean starch and trehalose of algal fronds (about $1 \mathrm{~g}$ fresh weight) fed with ${ }^{14} \mathrm{C}$-floridoside $\left(80 \mathrm{mg}\right.$ with $6.3 \times 10^{6} \mathrm{cpm}$ ) in the light or in the dark.

-
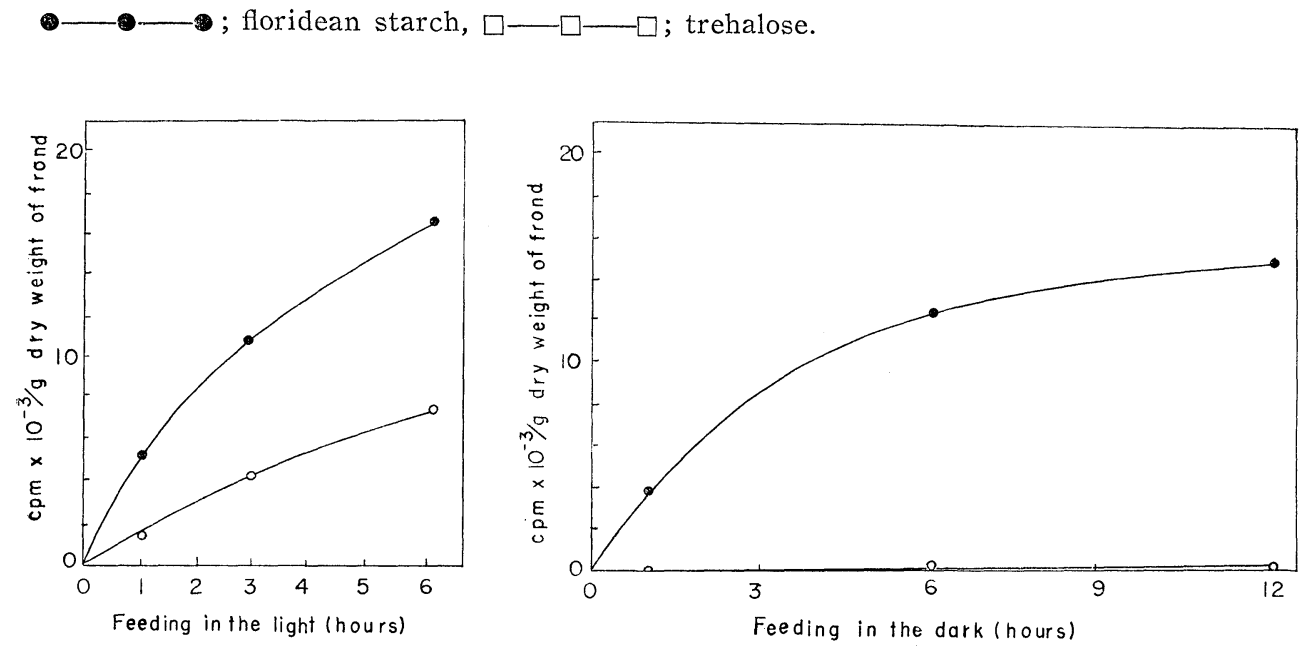

Fig. 6. Incorporation of radiocarbon into floridean starch and floridoside of algal fronds (about $1 \mathrm{~g}$ fresh weight) fed with ${ }^{14} \mathrm{C}$-trehalose $\left(60 \mathrm{mg}\right.$ with $2.5 \times 10^{6} \mathrm{cpm}$ ) in the light or in the dark.

; floridean starch, $\bigcirc-\bigcirc-O$; floridoside. 
Furthermore a smaller but significant amount of radiocarbon was incorporated into floridean starch of the algal fronds when they were fed with ${ }^{14} \mathrm{C}$-glycerol during illumination or in darkness, as shown in Table 5.

The results from Table 4 and Figs. 5 and 6 suggest that floridoside, trehalose and floridean starch are interconvertible storage products. However, the incorporation of radiocarbon into starch attained at most $1 \%$ of initial activity supplied to a feeding medium. This low incorporation may be due to low penetration of radiocarbon into algal cells; roughly $4 \%$ of the total counts supplied was found in the fronds with floridoside and trehalose, while nearly $14 \%$ with glycerol. However, an additional cause of this low incorporation into starch may be the possible consumption by respiration of ${ }^{14} \mathrm{C}$-substances which had been once taken up into cells.

Therefore, chopped algal fronds were fed with ${ }^{14} \mathrm{C}$-floridoside, ${ }^{14} \mathrm{C}$-trehalose or ${ }^{14} \mathrm{C}$-glycerol in the light or in the dark, and it was examined whether these ${ }^{14} \mathrm{C}$ substances are used for the respiration of cells. If this is the case, ${ }^{14} \mathrm{CO}_{2}$ would be produced from these ${ }^{14} \mathrm{C}$-compounds whithin the cells, and subsequently some of it might be deposited in cell wall as calcium carbonate. On this assumption, ${ }^{14} \mathrm{C}$ activity in calcium carbonate of the fronds was measured after feeding of the fronds with these ${ }^{14} \mathrm{C}$-carbohydrates. The results are shown in Table 6 . It is clear that the calcium carbonate was labelled by any of these three ${ }^{14} \mathrm{C}$-compounds to a high

Table 5. Incorporation of radiocarbon into floridean starch of algal fronds (about $1 \mathrm{~g}$ fresh weight) fed ${ }^{14} \mathrm{C}$-glycerol $\left(7.2 \mathrm{mg}\right.$ with $\left.4.9 \times 10^{5} \mathrm{cpm}\right)$ in the light or in the dark.

\begin{tabular}{lcc}
\hline Condition & Feeding time $(\mathrm{min})$ & $\begin{array}{c}\text { Radioactivity in floridean starch } \\
\left(\mathrm{cpm} \times 10^{-3} / \mathrm{g} \text { dry } \text { weight of frond }\right)\end{array}$ \\
\hline Light & 15 & 0.08 \\
& 30 & 0.34 \\
Dark & 60 & 1.04 \\
& 60 & 0.83 \\
\hline
\end{tabular}

Table 6. Incorporation of radiocarbon into $\mathrm{CaCO}_{3}$ of algal fronds fed ${ }^{14} \mathrm{C}$-compounds in the light or in the dark.

\begin{tabular}{lccc}
\hline \multirow{2}{*}{ Fed with } & Incubation period & \multicolumn{2}{c}{$\begin{array}{r}\text { Radioactivity in } \mathrm{CaCO}_{3}\left(\mathrm{cpm} \times 10^{-3} /\right. \\
\text { g dry weight of frond })\end{array}$} \\
\cline { 3 - 4 } & & 47.0 & 52.5 \\
& 1 (hour) & 21.0 & - \\
\hline${ }^{14}$ C-floridoside & 3 & 57.0 & 88.3 \\
$\left(80 \mathrm{mg}\right.$ with $\left.6.3 \times 10^{6} \mathrm{cpm}\right)$ & 6 & - & 97.9 \\
& 12 & 43.1 & 66.8 \\
${ }^{14} \mathrm{C}$-trehalose & 1 (hour) & 53.5 & - \\
$\left(60 \mathrm{mg}\right.$ with $\left.2.5 \times 10^{6} \mathrm{cpm}\right)$ & 3 & 78.0 & 79.4 \\
& 6 & - & 98.6 \\
${ }^{14} \mathrm{C}$-glycerol & 12 & 62.9 & - \\
$\left(7.2 \mathrm{mg}\right.$ with $\left.4.9 \times 10^{5} \mathrm{cpm}\right)$ & 15 (min) & 48.3 & - \\
& 30 & 52.1 & 68.3 \\
\hline
\end{tabular}


extent and the radioactivity increased with incubation time irrespective of the presence or the absence of light. These results support the above assumption that some portions of these ${ }^{14} \mathrm{C}$-substances taken up into algal cells may be consumed by the respiration of the cells.

\section{Discussion}

It was reported in a previous work ${ }^{8)}$ that floridoside and trehalose as well as floridean starch are the most abundant carbohydrates in S. maxima. In the present work, these carbohydrates were found to be rapidly labelled when the alga was illuminated in the presence of $\mathrm{H}^{14} \mathrm{CO}_{3}^{-}$in a culture medium. In particular, the radioactivity was most rapidly incorporated into floridoside (Fig. 2). The fact is consistent with the observation in other red algae ${ }^{4,5)}$. During the dark culture following 3 hour-illumination, however, the specific radioactivities and the amounts of floridoside and trehalose remained almost unchanged, while those of floridean starch were rapidly decreased, as clearly seen in the changes of their percent radioactivities. The fact, however, does not seem to mean that trehalose and floridoside are terminal storage products in view of the results from feeding experiments.

Namely, when the algal fronds were fed with ${ }^{14} \mathrm{C}$-floridoside or ${ }^{14} \mathrm{C}$-trehalose in the light, the radioactivities were incorporated not only from floridoside into treharose and vice versa, but also into floridean starch and calcium carbonate deposited in cell wall. This result shows first that these three kinds of carbohydrates are interconvertible with each other and secondly that they are partly metabolized through respiration. Therefore, the apparently unchanged radioactivities of floridoside and trehalose during the dark culture mentioned above suggest the existence of a metabolic pool among the these storage carbohydrates.

In feeding experiments using ${ }^{14} \mathrm{C}$-glycerol, the radioactivity was incorporated into floridean starch both in the light and in the dark in addition to its consumption for respiration. It may be therefore assumed that a part of glycerol is used for the synthesis of starch, possibly via reverse pathway of glycolysis.

In higher plants such as tobacco leaves, it was found that the amount of starch remarkably fluctuated during a day, but that of sucrose remained almost constant $t^{13,14)}$. This constancy of sucrose amount is effected by mutual convertibility between starch and sucrose. Floridoside in the present alga also behaves in a similar manner to sucrose in higher plants. This result verifies the assumption of Bean and Hassid ${ }^{3)}$ that a physiological role of floridoside in Iridophycus flaccidum would be similar to that of sucrose in higher plants.

Kauss $^{15)}$ has recently reported an interesting result that floridoside may be related to the maintenance of osmotic pressure in the cells of red algae. Such a role of floridoside is also similar to that of sucrose in higher plants. The constant concentration of floridoside and trehalose in S. maxima, therefore, seems to serve, in addition to a role of storage products, for the maintenance of the osmotic pressure of this alga.

On the other hand, experiments with ${ }^{14} \mathrm{CO}_{2}$ revealed that the radiocarbon was incorporated into trehalose more slowly than floridoside and floridean starch, and showed no remarkable change during the dark culture following illumination. Trehalose, therefore, seems to be slightly inert storage product as compared with the other two assimilation products.

In connection with these facts, a recent work of Majac, Craigie et al. ${ }^{4)}$ is very 
int eresting. They have reported that trehalose is the main photosynthetic product in a fresh water red alga, Batrachospermum sp., and it is also accumulated in Corallina officinalis in addition to floridoside ${ }^{5}$.

There has been no report on the metabolism of laminitol which is known to be distributed in some brown and red algae. This cyclitol was also found to be present in $S$. maxima, but it may have no significant meaning as a reserve substance, because very little radioactivity have been incorporated into this compound during illumination as compared with other soluble carbohydrates.

\section{References}

1) Lindberg, B., Second International Seaweed Symposium, 33 (Braarud, T., and S $\varnothing$ rensen, N.A., Ed., Pergamon Press, London, 1956).

2) Meeuse, B. J.D., Physiology and Biochemistry of Algae, 289 (Lewin, R. A., Ed., Academic Press, New York, 1962).

3) Bean, R.C., and Hassid, W.Z., J. Biol. Chem. 212: 411 (1955).

4) Majak, W., Craigie, J.S., and McLachlan, J., Can. J. Botany $44: 541$ (1966).

5) Craigie, J.S., McLachlan, J., and Tocher, R. D., Can. J. Botany 46: 605 (1968).

6) Meeuse, B. J.D., Andries, M., and Wood, J. A., J. Exp. Botany 11: 129 (1960).

7) Ozaki, H., Maeda, M., and Nisizawa, K., J. Biochem. (Tokyo) 61: 497 (1967).

8) Nagashima, H., Nakamura, S., and Nisizawa, K., Bot. Mag. (Tokyo) 82: 379 (1969).
9) Dubois, M., Gilles, K. A., Hamilton, J.K., Rebers, P. A., and Smith, F., Anal. Chem. 28 : 350 (1956).

10) Somogyi, M., J. Biol. Chem. 195: 19 (1952).

Nelson, N., J. Biol. Chem. 153: 375 (1944).

11) Peters, J.H., and Gutman, H.R., Anal. Chem. 25 : 987 (1953).

12) Turvey, J.R., and Simpson, P.R., Proceedings of the Fifth International Seaweed Symposium, 323 (Gordon Young, E., and McLachlan, J.L., Ed., Pergamon Press, Oxford, London, 1966).

13) Porter, H.K., Martin, R.V., and Bird, I. F., J. Exp. Botany 10: 264 (1959).

14) Madsen, E., Physiologia Plantarum 21: 168 (1968).

15) Kauss, H., Z. Pflanzenphysiol. 58: 428 (1968). 